\title{
Exoplanet bounty includes most Earth-like worlds yet
}

Latest crop from the Kepler telescope brings small planets around Sun-like stars - and an ice world.

\section{Alexandra Witze}

06 January 2015

\section{SEATTLE, WASHINGTON}

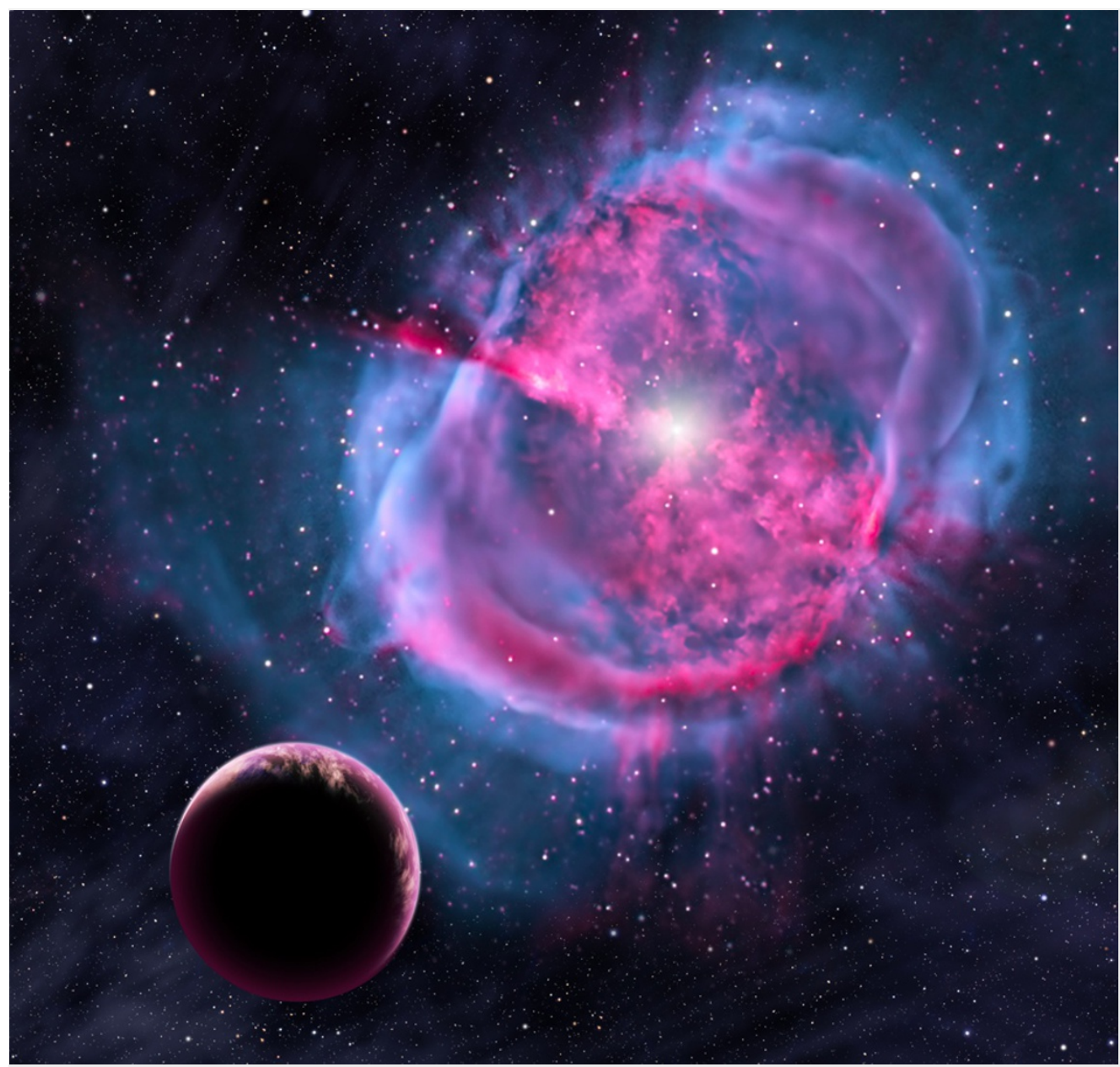

David A. Aguilar/CFA

Exoplanets have been discovered around hundreds of stars.

NASA's Kepler space telescope has hit a milestone: confirming the existence of 1,000 planets, including a fresh crop of alien worlds that are more Earth-like than any yet found.

The newfound planets include one with a radius that is just 1.1 times that of Earth, and receives more energy from its star than the previous best 'Earth twin' did. Scientists announced its existence on 6 January in Seattle, Washington, at a meeting of the American Astronomical Society.

"We're really increasing the population of these special planets that Kepler was designed to find," says team member Douglas Caldwell, an astronomer at the SETI Institute in Mountain View, California.

Using an additional year's worth of observations from Kepler, other astronomers reported finding 554 new planetary candidates. These still need to be confirmed as actual planets and not just data blips, but many of them appear to orbit relatively Sun-like stars.

Together, the findings help to nudge astronomers closer to Kepler's ultimate goal: calculating the frequency of planets around nearby stars. "We're not only homing in on Earth-like things, but also filling in the Earth-like population," says Caldwell. 


\section{Data dump}

The spacecraft's planet-hunting job is essentially over, after the failure in May 2013 of the second of two of the reaction wheels that help the telescope to orient itself. Kepler continues to explore the Universe in a limited 'K2' mission, which observes for only short periods of time.

But there are enough data from the four years that Kepler operated properly, between 2009 and 2013, to discover new planets. Competing teams have been sifting through the observations using different techniques, and then using additional telescopes to follow up the most interesting candidates.

Caldwell's team, led by Guillermo Torres of the Harvard-Smithsonian Center for Astrophysics in Cambridge, Massachusetts, worked with three years of Kepler data. The researchers drew up a long list of planet candidates that are thought to be no more than twice the size of Earth, and to orbit within the habitable zones of stars - that is, at the right distance from the star for the surface temperature to support liquid water.

Torres then used a computer program to calculate the statistical likelihood that the planetary candidates are in fact real planets. The team ended up with eight new planets in habitable zones.

"People want to know what is the most Earth-like," says Caldwell. Two of the eight planets, Kepler-438b and Kepler-442b, fit that category. Both are just a little bit bigger than Earth and are warmed by their stars more than previous Earth-twin candidates. One of the planets circles a cool star known as an M-dwarf, while the second circles a slightly hotter star. (The brighter the star, the more energy that can reach the planet's surface and potentially support life.)

\section{Hide and seek}

Other research suggests that M-type stars may not host quite as many planets as scientists had thought. In preliminary work presented at the Seattle meeting, Christopher Burke of the SETI Institute analysed the rate at which Kepler has been finding planets around stars of different temperatures.

Planets with short periods appeared more frequently around M-type stars than they did around warmer stars, Burke found. But he discovered no M-type stars with planets that take longer than 150 days to orbit. "I was shocked," he said.

It is not entirely clear what that absence might mean. But Burke's work shows how Kepler's discoveries are allowing astronomers to put much better numbers on exactly where and when planets do appear.

All four years of Kepler data are now available online, reported Fergal Mullally of NASA's Ames Research Center in Moffett Field, California. His analysis turned up the more than 500 new planetary candidates.

They include a possible planet that is between 0.8 and 1.8 times the size of Earth, which orbits a Sun-like star every 376 days tantalizingly close to Earth's 365-day period. Other candidates include three Earth-sized planets in a single planetary system, and another that orbits far beyond its star, making it an 'ice world' like the planet Hoth in Star Wars.

"There's a lot of really exciting science to come out of Kepler for a really long time," says Angie Wolfgang, an astronomer at the University of California, Santa Cruz.

Nature I doi:10.1038/nature.2015.16673 